\title{
MODELADO Y CONTROL DE UN VEHÍCULO SUBACUÁTICO NO TRIPULADO
}

\author{
Francisco J. Velasco González \\ Univ. Cantabria. E.T.S. de Náutica, C/ Gamazo 1- 39004 Santander (Cantabria), velascof@unican.es \\ Elías Revestido Herrero \\ Univ. Cantabria. E.T.S. de Náutica, C/ Gamazo 1- 39004 Santander (Cantabria), revestidoe@unican.es \\ Francisco Javier Lastra Santos \\ Univ. Cantabria. E.T.S. de Náutica, C/ Gamazo 1- 39004 Santander (Cantabria), fjlastra@teisa.unican.es \\ Luis Manuel Vega Antolín \\ Univ. Cantabria. E.T.S. de Náutica, C/ Gamazo 1- 39004 Santander (Cantabria), vegalm@unican.es
}

\section{Resumen}

En el presente artículo, basándonos en un conjunto específico de ensayos llevados a cabo en el Canal de Experiencias Hidrodinámicas del Pardo CEHIPAR, se realiza una estimación de los parámetros hidrodinámicos de un vehículo subacuático con forma de torpedo. Además, se propone una modificación del método LOS convencional que funciona correctamente en presencia de corrientes oceánicas. El LOS modificado se aplica al modelo en forma de torpedo obtenido previamente y se prueba en condiciones realistas.

Palabras Clave: PMM, estimación de parámetros, LOS, seguimiento de camino, corrientes oceánicas.

\section{INTRODUCCIÓN}

El uso de vehículos no tripulados, en el campo naval, es ampliamente conocido en el mundo científico. Los sectores militares y de seguridad son los que se están tirando de esta tecnología en los últimos años. la formación de la flota constituye uno de los requisitos básicos para el diseño de una nueva generación de vehículos submarinos que se emplearán en diversas misiones, como detección de minas, guerra antisubmarina, perímetro de defensa, guerra de superficie, el apoyo a las fuerzas de operaciones especiales, etc.

Hoy en día, los AUV-UUVs son de suma importancia, tanto para aplicaciones y procedimientos de defensa como para aplicaciones civiles para la exploración submarina. La incorporación de los vehículos no tripulados para el sector de Defensa han contribuido al estado de la técnica de los sistemas no tripulados [14] para misiones peligrosas o de alto riesgo, como el seguimiento, detección y neutralización de minas.

Resulta de gran importancia, en construcción naval, obtener un modelo matemático de maniobra lo más exacto que sea posible. Este requisito es también de suma importancia en aplicaciones de control de movimiento en el que, si el modelo matemático utilizado para el diseño de control no es exacto cuando se consideran las condiciones de funcionamiento del vehículo, o si existen perturbaciones externas, es difícil ajustar el controlador para una buen comportamiento del vehículo. Una manera de obtener los parámetros de un modelo de maniobra es llevar a cabo pruebas de tipo PMM [8] Hay algunos artículos relacionados con las pruebas realizadas en PMM con vehículos submarinos [7,10]. En este trabajo, hemos hecho algunas modificaciones en las pruebas PMM con el fin de estimar los coeficientes de balance y los parámetros de los actuadores.

En base a los citados modelos de maniobra se desarrollan sistemas de seguimiento de camino. Line of Sight (LOS) es un método ampliamente conocido de navegación que proporciona resultados satisfactorios para el seguimiento de un camino definido por waypoints $[1,6,11,12,13]$. Sin embargo, este método tiene la desventaja de que si el vehículo está funcionando en condiciones realistas, tales como en presencia de corrientes, se pueden producir grandes errores transversal de ruta. En este caso, no hay un número tan grande de publicaciones tanto como en el método convencional LOS y las contribuciones existentes que tienen que ver con las corrientes oceánicas son bastante complejos como en Caharija [2,3]. En este trabajo, se propone una modificación del método LOS convencional que proporciona resultados satisfactorios en presencia de corrientes oceánicas mediante la realización de un procedimiento sencillo.

\section{MODELO MATEMÁTICO}


Los vehículos submarinos se mueven en seis grados de libertad (GDL). Con el fin de describir el movimiento del vehículo, se necesitan tres coordenadas de traslación y otras tres para definir la orientación. Se utilizan habitualmente, dos sistemas de coordenadas para estudiar el movimiento del vehículo: uno fijo al vehículo para definir su traslación y movimientos de rotación y el otro se encuentra en Tierra para describir su posición y orientación.

El modelo no lineal de maniobra de 6 GDL puede expresarse en la forma siguiente $[4,5]$ :

$$
\begin{aligned}
& M \dot{v}+C(v) v+D(v) v+g(\eta)=\tau \\
& y=\eta+w, \dot{\eta}=R(\eta) v,
\end{aligned}
$$

donde $\eta=[x, y, z, \phi, \theta, \psi]^{T}$ es el vector de posición $\mathrm{y}$ ángulos de Euler, $v=[u, v, w, p, q, r]^{T}$ son las velocidades lineales y angulares, $v=[X, Y, Z, K, M, N]^{T}$ a son las fuerzas y momentos y $w$ es el ruido en la medición. $\boldsymbol{M}$ es la matriz de masa adicional y cuerpo rígido, $C(v) v$ es el término de Coriolis y Centripeto, $g(\eta)$ es la matriz de restauración y $R(\eta)$ es la matriz de rotación y $D(v) v$ representa las fuerzas de amortiguación hidrodinámicos que son una combinación de términos lineales y no lineales de amortiguación. Las matrices de la ecuación (1) considerando las simetrías del vehículo se pueden encontrar en Fossen [5].

En el presente trabajo, se utiliza un vehículo con forma de torpedo, que incorpora tres motores: dos horizontales situados en el centro del vehículo para el movimiento de guiñada y una vertical para el control de profundidad.

El empuje proporcionado por una hélice se puede expresar como:

$$
T=\rho D_{h}^{4} K_{T}\left(j_{0}\right) n|n|(1-t)
$$

donde $\rho$ es la densidad del agua, $D_{h}$ es el diámetro de la hélice, $n$ son las revoluciones por segundo de la hélice, $t$ es el factor de deducción de empuje (valores típicos de 0.05 a 0.2 ) y $K_{T}$ es un coeficiente adimensional [4]. En base a la ecuación de empuje (2) y el momento de guiñada, la siguiente expresión se obtiene según Refsnes [13]:

$$
\left[\begin{array}{c}
\tau_{x, t h} \\
\tau_{N, t h}
\end{array}\right]=\left[\begin{array}{cc}
1 & 1 \\
d_{p} & -d_{p}
\end{array}\right]\left[\begin{array}{c}
T_{p} \\
T_{s}
\end{array}\right]
$$

donde $\tau_{x, t h}$ es la fuerza de avance, $\tau_{N, t h}$ es el momento de guiñada, $d_{p}$ la distancia desde el centro de la masa a la hélice, $T_{p}$ es el empuje de estribor y $T_{s}$ es el empuje de babor. Además, si el ángulo de balance $\Phi \neq 0$, la siguiente ecuación se ha de tener en cuenta:

$$
\tau_{M, t h}=-\left(T_{p}-T_{s}\right) d_{p} \sin (\phi)
$$

As a result, the forces generated by the thrusters and the pitch actuator are $\tau=\left[\tau_{X}, 0, \tau_{Z}, 0, \tau_{M}, \tau_{N}\right]^{T}$.

\section{ESTIMACIÓN DE PARAMETROS}

En esta sección se resumen las pruebas que se realizaron en el "Canal de Experiencias Hidrodinámicas de El Pardo" CEHIPAR para la estimación de parámetros del modelo matemático definido anteriormente. Para ello, hemos utilizado un vehículo con forma de torpedo, que tiene una longitud máxima de $1,65 \mathrm{~m}$ y un radio de $0,17 \mathrm{~m}$.

La figura 5 ilustra el montaje del vehículo C'Inspector en las instalaciones del CEHIPAR. El conjunto de la Figura 5 tiene una mesa de medición, en el que se monta dos cilindros que sostienen el vehículo. Estos cilindros son comandados por los motores eléctricos. Los cilindros son del tipo de tornillo, para proporcionar una alta precisión en el movimiento. La mesa de medición se monta en un sistema que se mueve a lo largo del canal de experiencias CEHIPAR.

Antes de todos estos ensayos, fue necesario determinar los momentos de inercia, el centro de gravedad y el centro de flotabilidad del vehículo. Los valores obtenidos se encuentran en la Tabla 2, que incluye los valores de peso con y sin el peso de la seguridad.

Tabla 1 Distribución de pesos del vehículo con forma de torpedo con y sin el peso de la seguridad.

\begin{tabular}{|l|l|l|}
\hline & \multicolumn{2}{|l|}{ Peso de seguridad } \\
\hline & con & sin \\
\hline Peso $(\mathrm{Kg})$ & 33 & 34,34 \\
\hline $\mathrm{x}_{\mathrm{g}}(\mathrm{m})$ & 0,184 & 0,167 \\
\hline $\mathrm{Zg}_{\mathrm{g}}(\mathrm{m})$ & $-0,012$ & $-0,012$ \\
\hline $\mathrm{I}_{\mathrm{xx}}(\mathrm{kg} \mathrm{m} 2)$ & 0,287 & 0,29 \\
\hline $\mathrm{I}_{\mathrm{yy}}(\mathrm{kg} \mathrm{m} 2)$ & 7,105 & 6,945 \\
\hline $\mathrm{I}_{\mathrm{zz}}(\mathrm{kg} \mathrm{m} 2)$ & 7,233 & 7,073 \\
\hline
\end{tabular}

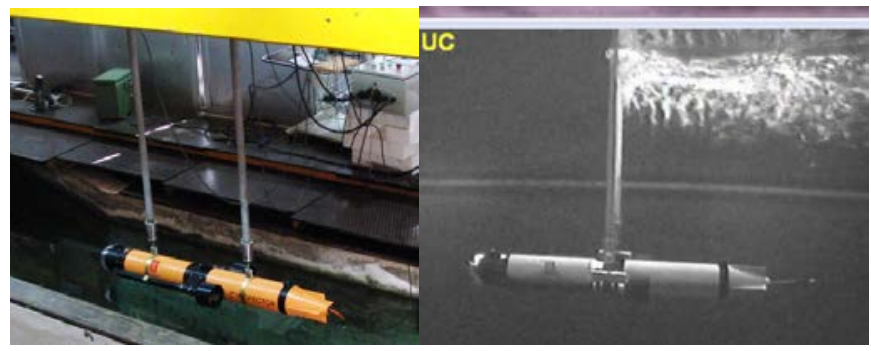

Fig. 1 Montaje del vehiculo con forma de torpedo C'Inspector en las instalaciones del CEHIPAR.

Todos los parámetros estimados en esta sección se refieren a un sistema de coordenadas ortogonal situado a una distancia de $785 \mathrm{~mm}$ del extremo de proa del vehículo (a la altura del orificio de fijación perno de ojo situado entre el propulsor vertical y el orificio de compensación). Los parámetros 
obtenidos en cada uno de los ensayos se han estimado por mínimos cuadrados ordinarios [9].

Dado que las aceleraciones de la ecuación (1) no se midieron, se ha aplicado un suavizador polinómica de punto fijo para estimarlas. Es decir, para cada medición de la velocidad se ajusta un polinomio local basada en una serie de mediciones cercanas a la medición de interés,

$$
\hat{v}_{j}\left(t_{k}\right)=\hat{a}_{0}+\hat{a}_{1} \Delta_{k, i}+\hat{a}_{1} \Delta+\hat{a}_{1} \Delta_{k, i}
$$

$$
\text { donde } \Delta_{k, i} \square t_{k+1}-t_{k} i=-M_{s}, \ldots,-1,0,1, \ldots, M_{s} \mathrm{y} j=1 \ldots 6
$$

(grados de libertad). Entonces, la medición suavizada y sus derivadas se obtienen tomando

$$
\hat{v}_{j}\left(t_{k}\right)=\hat{a}_{0}, \quad \hat{\dot{v}}_{j}\left(t_{k}\right)=\hat{a}_{1}
$$

La Figura 2 muestra los resultados del suavizador (ecuaciones 6 y 7) para el grado de libertad de avance. Se obtiene la fuerza $X$ filtrada y la velocidad $u$ filtrada y su derivada, ya que no se midió en los ensayos. El resto de los grados de libertad se filtran de la misma manera como en la Figura 2. En el diseño del suavizador, se ha considerado el compromiso entre sesgo y la varianza, como se explica en Wasserman [16]. Es decir, si los datos están lo suficientemente suavizados, el resultado es datos con poco sesgo y varianza grande; pero si por el contrario el suavizado es elevado, los datos resultantes son con variación pequeña y gran sesgo. El parámetro $M_{s}$ controla este compromiso entre la varianza y el sesgo.

En la tabla 2 se resumen las pruebas PMM que se llevaron a cabo para estimar los parámetros del modelo definido en el apartado 2. Adicionalmente a las pruebas convencionales PMM que estiman los parámetros de balance y guiñada como en Phillips [10] o de arfada y cabezada como en Guo [7], hemos realizado una prueba específica para estimar los parámetros de balance. El montaje de la prueba de balance está en la imagen de la derecha de la figura 1.

Por otra parte, se han realizado dos pruebas diferentes para estimar los parámetros de las unidades de propulsión horizontal y vertical. En ambos casos, el vehículo ha sido arrastrado a la velocidad de $1,5 \mathrm{~m} / \mathrm{s}$, mientras que las revoluciones de las hélices varían entre $35 \%$ y $60 \%$ de las revoluciones máximas de las unidades de propulsión horizontal y entre $-60 \%$ y $60 \%$ de la revoluciones máximas de la unidad de propulsión vertical.
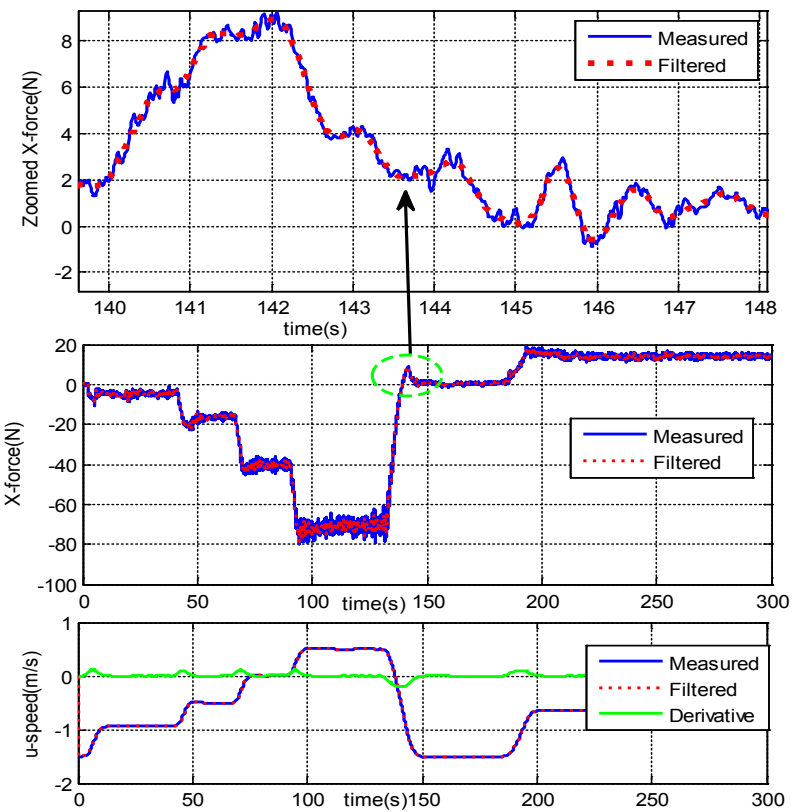

Fig. 2 Resultados del suavizador para el grado de libertad de

\begin{tabular}{|c|c|c|}
\hline Ensayo & Ecuaciones & Amplitudes/Velocidades/Revoluciones \\
\hline $\begin{array}{l}\text { Resistencia y aceleración } \\
\text { longitudinal }\end{array}$ & $X=X_{0}+X_{u} u+X_{u|u|} u|u|+\left(-m+X_{\dot{u}}\right) \dot{u}$ & $(0.5 \mathrm{~m} / \mathrm{s}-2.0 \mathrm{~m} / \mathrm{s})$ \\
\hline $\begin{array}{l}\text { Cabezada estática y } \\
\text { dinámica }\end{array}$ & $\begin{array}{l}w=U \sin \theta, \quad Z=Z_{w} w, \\
Z=Z_{\dot{q}} \dot{q}+\left(Z_{q}-m U\right) q \\
M=\left(-I_{y y}+M_{\dot{q}}\right) \dot{q}+\left(M_{q}-m x_{g} U\right) q\end{array}$ & $\left(5^{\circ}\right.$ and $\left.10^{\circ}\right)$, periodo de oscilación $3 \mathrm{~s}$. \\
\hline Arfada dinámica & $\begin{array}{l}Z=\left(-m+Z_{\dot{w}}\right) \dot{w}+Z_{w|w|} w|w|+Z_{w} w \\
M=M_{\dot{w}} \dot{w}+M_{w} w\end{array}$ & $\begin{array}{l}(0.05 \mathrm{~m}-0.15 \mathrm{~m}), \text { periodo de oscilación } 3 \\
\text { s. }\end{array}$ \\
\hline Guiñada estática y dinámica & $\begin{array}{l}u=U \sin \psi, Y=Y_{v} v, N=N_{v} v, \\
Y=Y_{\dot{r}} \dot{r}+\left(Y_{r}-m U\right) r+Y_{r|r|} r|r| \\
N=\left(-I_{z z}+N_{\dot{r}}\right) \dot{r}+\left(N_{r}-m x_{g} U\right) r\end{array}$ & $\left(5^{\circ}\right.$ to $\left.10^{\circ}\right)$ \\
\hline
\end{tabular}
avance.

Table 2. Conjunto de ensayos llevados a cabo en las instalaciones del CEHIPAR. 


\begin{tabular}{|l|l|l|}
\hline $\begin{array}{l}\text { Desplazamiento lateral } \\
\text { dinámico }\end{array}$ & $Y=\left(-m+Y_{\dot{v}}\right) \dot{v}+Y_{v|v|} v|v|+Y_{v} v$ & $(0.05 \mathrm{~m}-0.15 \mathrm{~m})$ \\
\hline Balance dinámico & $K=\left(-I_{x x}+K_{\dot{p}}\right) \dot{p}+K_{p} p$ & $\begin{array}{l}(10,20 \text { and 30), periodo de oscilación 3 } \\
\mathrm{s} .\end{array}$ \\
\hline Propulsión horizontal & Equation 3. & $\begin{array}{l}35 \% \text { y } 60 \% \text { de las revoluciones } \\
\text { máximas. }\end{array}$ \\
\hline Propulsión vertical & Equation 3. & $\begin{array}{l}-60 \% \text { y } 60 \% \text { de las revoluciones } \\
\text { máximas. }\end{array}$ \\
\hline Actuador de cabezada & $\begin{array}{l}M=M_{p} . d, \text { donde } d \text { es el tanto por ciento de } \\
\text { desplazamiento, Hay que señalar que este } \\
\text { momento debe ser añadido a la } \\
\tau_{M} .\end{array}$ & $\begin{array}{l}0 \% \text { y 100\% del desplazamiento } \\
\text { máximo. }\end{array}$ \\
\hline
\end{tabular}

El vehículo con forma de torpedo está equipado con un actuador de cabezada, que consiste en el desplazamiento de una masa interna. Para la estimación de los parámetros de este actuador, se llevó a cabo un ensayo con el vehículo en una posición fija mientras que la masa interna se mueve entre el $0 \%$ y el $100 \%$ del desplazamiento máximo (véase la última fila de la tabla 2).

\section{1 VALIDACIÓN DEL MODELO}

En la aplicación de la teoría de identificación de sistemas, es habitual utilizar para la validación del modelo datos diferentes de los datos utilizados en la estimación. En este trabajo, por razones económicas, a fin de evitar un gran número de ensayos, la mitad de los datos adquiridos se utilizaron para la estimación de parámetros y la otra mitad para la validación del modelo. Hay diferentes maneras de validar un modelo [9], uno de ellos es el uso de métricas estadísticas. Una métrica es el coeficiente de determinación $R^{2}(\%)$, que proporciona información sobre el modelo obtenido en la medida en que es capaz de reproducir los datos de medición, y representa el porcentaje de variación de salida reproducida por el modelo:

$$
R^{2}(\%)=\frac{\sum(\widehat{D}-\bar{D})^{2}}{\sum(\widehat{D}-D)^{2}+\sum(\widehat{D}-\bar{D})^{2}}
$$

donde D y $\bar{D}$ representa los datos medidos y su media respectivamente, y $\hat{D}$ los datos generados por el modelo obtenido.

La Tabla 3 muestra el resumen de los resultados de la validación del modelo, mediante el cálculo del coeficiente de determinación, para cada una de las pruebas realizadas. Por lo tanto, se concluye que el modelo obtenido es bueno, dado que en la mayoría de los ensayos el coeficiente $R^{2}(\%)$ es elevado. Debe de tenerse en cuenta que el coeficiente de $R^{2}(\%)$ obtenido también depende de la relación medida de señal/ruido correspondiente a la prueba. Esto sucede, en el ensayo de cabezada dinámico ya que la relación señal/ruido es alto y por lo tanto el coeficiente de $R^{2}(\%)$ es más pequeño que el resto de los grados de libertad.

Table 3 Resultados del coeficiente de determinación para los ensayos de 6GDL.

\begin{tabular}{|l|l|l|}
\hline Ensayo & Fuerzas & $R^{2}(\%)$ \\
\hline $\begin{array}{l}\text { Resistencia y aceleración } \\
\text { longitudinal }\end{array}$ & $\mathrm{X}(\mathrm{N})$ & 69,23 \\
\hline Cabezada dinámica & $\mathrm{Z}(\mathrm{N})$ & 47,93 \\
\hline Arfada dinámica & $\mathrm{Z}(\mathrm{N})$ & 99,24 \\
\hline Guiñada dinámica & $\mathrm{Y}(\mathrm{N})$ & 70,47 \\
\hline $\begin{array}{l}\text { Desplazamiento Lateral } \\
\text { dinámico }\end{array}$ & $\mathrm{Y}(\mathrm{N})$ & 96,86 \\
\hline & $\mathrm{Momento}$ & \\
\hline Balance dinámico & $\mathrm{K}(\mathrm{Nm})$ & 85,55 \\
\hline & & \\
\hline
\end{tabular}

\section{CONTROL DEL VEHICULO}

En esta sección se propone una modificación del algoritmo LOS convencional con el fin de reducir los errores de camino transversal en presencia de corrientes oceánicas. El LOS modificado implica el diseño de un controlador de rumbo basado en el modelo obtenido en la sección anterior. El controlador de rumbo utilizado en este trabajo se basa en un trabajo previo [15], donde un controlador de red de primer orden, que evita el problema de wind up, se ajusta basándose en algoritmos genéticos. 


\subsection{ALGORITMO LOS MODIFICADO}

El LOS modificado conlleva la realización de los siguientes pasos:

\section{Inicialización}

Establecer los valores iniciales de las constantes y variables que intervienen en el método: $X_{w p}, Y_{w p}, i, j, c$, $n_{w}, n, n_{s}$.

\section{Way point $i=1$}

$$
\psi_{\text {dini }}=\operatorname{arctg}\left(\frac{Y_{w p}(1)-Y_{w p}(0)}{X_{w p}(1)-X_{w p}(0)}\right)
$$

Dividir la línea definida por waypoints $(i, i-1)$ en $n_{p}$ puntos.

$$
\mathrm{D}_{\mathrm{x}}=\left(X_{w p}(1)-X_{w p}(0)\right) / n_{p}
$$$$
\mathbf{j}=\mathbf{1}
$$

do

$$
\begin{aligned}
& \psi_{d}(t)=\operatorname{arctg}\left(\frac{\operatorname{tg}\left(\psi_{\text {dini }}\right) \cdot j \cdot D_{x}+Y_{w p}(0)-y(t)}{X_{w p}(0)+j \cdot \operatorname{dist}-x(t)}\right) \\
& n_{s}=n_{s}+1, t=n_{s} T
\end{aligned}
$$

While $x(t) \leq X_{w p}(0)+j \cdot D_{x}$

\section{$\mathbf{j}=\mathbf{n}$}

do

$$
\begin{aligned}
& \psi_{d}(t)=\operatorname{arctg}\left(\frac{\operatorname{tg}\left(\psi_{\text {dini }}\right) \cdot j \cdot D_{x}+Y_{w p}(0)-y(t)}{X_{w p}(0)+j \cdot \operatorname{dist}-x(t)}\right) \\
& n_{s}=n_{s}+1, t=n_{s} T
\end{aligned}
$$

While

$$
\begin{aligned}
& x(t) \leq X_{w p}(0)+j \cdot D_{x} \& \\
& \left(X_{w p}(i)-x(t)\right)^{2}+\left(Y_{w p}(i)-y(t)\right)^{2} \leq\left(c \cdot L_{p p}\right)^{2} \\
& \mathrm{i}=\mathrm{i}+1
\end{aligned}
$$

\section{Way point $i=n_{w}$}

$$
\psi_{\text {dini }}=\operatorname{arctg}\left(\frac{Y_{w p}\left(n_{w}\right)-Y_{w p}\left(n_{w}-1\right)}{X_{w p}\left(n_{w}\right)-X_{w p}\left(n_{w}-1\right)}\right)
$$

Dividir la línea definida por waypoints $(i, i-1)$ en $n_{p}$ puntos.

$$
D_{x}=\left(X_{w p}\left(n_{w}\right)-X_{w p}\left(n_{w}-1\right)\right) / n_{p}
$$

\section{$\mathrm{j}=\mathbf{1}$}

do

$$
\psi_{d}(t)=\operatorname{arctg}\left(\frac{\operatorname{tg}\left(\psi_{d i n i}\right) \cdot j \cdot D_{x}+Y_{w p}(0)-y(t)}{X_{w p}(0)+j \cdot \operatorname{dist}-x(t)}\right)
$$

$$
n_{s}=n_{s}+1, t=n_{s} T
$$

While $x(t) \leq X_{w p}(0)+j \cdot D_{x}$

$\mathbf{j}=\mathbf{n}$

do

$$
\begin{aligned}
& \psi_{d}(t)=\operatorname{arctg}\left(\frac{\operatorname{tg}\left(\psi_{\text {dini }}\right) \cdot j \cdot D_{x}+Y_{w p}(0)-y(t)}{X_{w p}(0)+j \cdot \operatorname{dist}-x(t)}\right) \\
& n_{s}=n_{s}+1, t=n_{s} T
\end{aligned}
$$

While $x(t) \leq X_{w p}(0)+j \cdot D_{x}$

donde $X_{w p}$ y $Y_{w p}$ son los vectores en el eje $x$ e $y$ correspondientes a 1 waypoints a seguir por el vehículo, $n_{w}$ el número de vectores puntos de ruta, $n$ el número de puntos en los que se divide la línea entre $i$ e $i-1, c$ el tamaño de la círculo de aceptación, $n_{s}$ es el número de muestras y $T$ es el tiempo de muestreo. El valor de $n$ puede ser seleccionado teniendo en cuenta el tipo de misión que cumplir y la distancia entre los waypoints, se adopta como una regla de oro $n_{p}=a b s\left(X_{w p}(i)-X_{w p}(i-1)\right) / L_{p p}$ siendo $\mathrm{L}_{\mathrm{pp}}$ la longitud del vehículo en forma de torpedo.

\section{SIMULACIÓN DE RESULTADOS}

Las figuras 3-6 muestran simulaciones implementadas en el entorno Matlab/Simulink. Se ha implementado el modelo definido en el apartado 2 con los parámetros estimados (sección 3) y un sistema de seguimiento basado en el método LOS modificado establecido en el apartado 4. Los resultados muestran una reducción en el error transversal de camino cuando se aplica el método LOS modificado como se puede ver mediante la comparación de la Figura 3 y 4 sin corrientes oceánicas. Las figuras 5 y 6 , bajo una corriente oceánica de $0,3 \mathrm{~m} / \mathrm{s}$, pone de manifiesto que el 
método LOS modificado es capaz de seguir la trayectoria definida por los waypoints mientras que el método LOS convencional no es capaz.

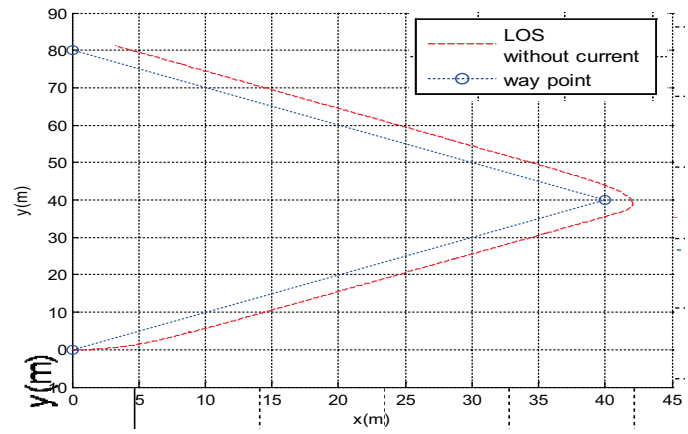

Fig. 3 Resultados de seguimiento para el método LOS convencional sin corriente.

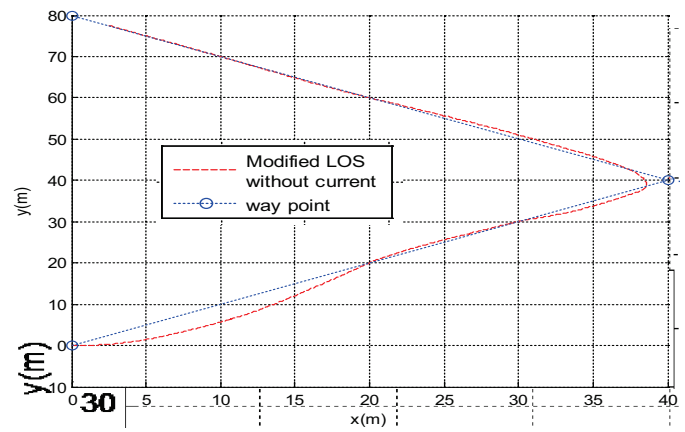

Fig. 4 Resultados de seguimiento para el método LOS modificado sin corriente.

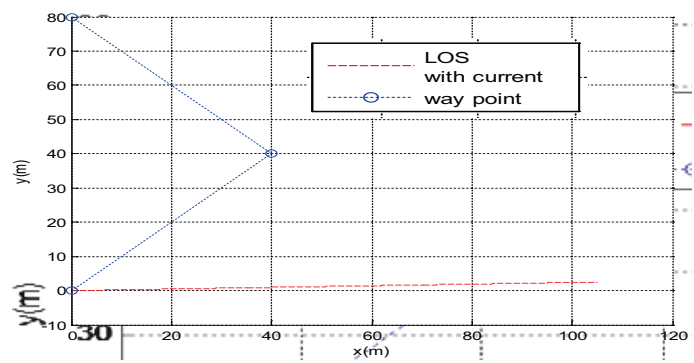

Fig. 5 Resultados de seguimiento para el método LOS convencional con corriente $(0.3 \mathrm{~m} / \mathrm{s})$.

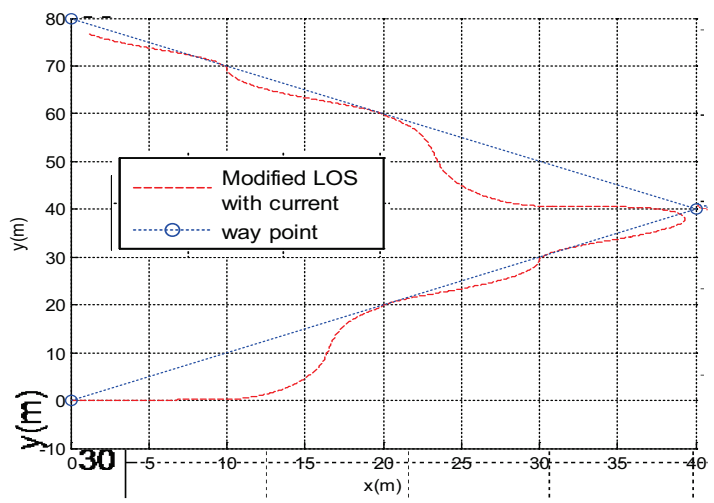

Fig. 6. Resultados de seguimiento para el método LOS modificado con corriente $(0.3 \mathrm{~m} / \mathrm{s})$.

\section{CONCLUSIONES}

En este trabajo, se ha puesto de manifiesto el estudio en términos de modelado de un vehículo submarino no tripulado a través de un programa de ensayos en las instalaciones del CEHIPAR. Gracias a los datos adquiridos, ha sido posible estimar los coeficientes hidrodinámicas de un modelo no lineal de maniobra de seis grados de libertad de un vehículo submarino con forma de torpedo incluyendo los coeficientes correspondientes a los actuadores.

Además, se ha propuesto una modificación del método LOS convencional que proporciona resultados adecuados bajo corrientes oceánicas en base al modelo obtenido con los datos del CEHIPAR.

\section{Agradecimientos}

Este proyecto ha sido parcialmente financiado por el Ministerio de Defensa español (programa1003211003100 COINCIDENTE) y por el MINECO: DPI 2.011-27.990 con fondos FEDER.

\section{Referencias}

[1] Aguiar A. and Pascoal A., (1997) "Modeling and control of an autonomous underwater shuttle for the transport of benthic laboratories," in Proc. of MTS/IEEE Conference OCEANS '97. , pp. 888-895.

[2] Caharija, W.; Pettersen, K.Y.; Gravdahl, J.T.; Borhaug, E., (2012 a) "Integral LOS guidance for horizontal path following of underactuated autonomous underwater vehicles in the presence of vertical ocean currents," American Control Conference (ACC), vol., no., pp.5427,5434, 2729

[3] Caharija, W.; Pettersen, K.Y.; Gravdahl, J.T.; Borhaug, E., (2012 b) "Path following of underactuated autonomous underwater vehicles in the presence of ocean currents," Decision and Control (CDC), 2012 IEEE 51st Annual Conference on , vol., no.,pp.528,535,10-13Dec.

[4] Fossen, T.I. (1994). Guidance and control of ocean vehicles. USA: Wiley, New York.

[5] Fossen, T. I. (2002). Marine control systems: Guidance, navigation and control of ships, rigs and underwater vehicles. Marine Cybernetics AS, Trodheim, Norway.

[6] Fossen T. I., Breivik M., and Skjetne R., (2003) "Line-of-Sight path following of underactuated marine craft," in Proc. of the 6th IFAC Conference on Manoeuvring and Control of Marine Craft, Girona, Spain, pp. 244-249.

[7] Guo, J., Chiu, F.: Maneuverability of a Flatstreamlined Underwater Vehicle. ;In ICRA(2001)897-902 
[8] Lewis, V. (1998). Principles of Naval Arquitecture. The Society of Naval Architects and Marine Engineers.

[9] Ljung, L. (1999). System identification: Theory for the user (Second edition). Upper Saddle River, Prentice Hall Inc. New Jersey.

[10] Phillips, A.B., Furlong, M. and Turnock, S.R. (2007) Virtual planar motion mechanism tests of the autonomous underwater vehicle autosub. In, STG-Conference / Lecture day "CFD in Ship Design", Hamburg, Germany, 26 Sep 2007. Hamburg, Germany, Institute M-8 of Hamburg University of Technology8pp.

[11] Healey A. and Lienard D., (1993) "Multivariable sliding mode control for autonomous diving and steering of unmanned underwater vehicles," IEEE Journal of Oceanic Engineering, vol. 18, no. 3, pp. 327-339, July.

[12] Pettersen K. and Lefeber E., (2001) "Waypoint tracking control of ships," in Proc. of the 40th IEEE Conference on Decision and Control, pp. 940-945.

[13] Refsnes, J.E.; Sorensen, (2004) A.J., "Design of control system of torpedo shaped ROV with experimental results," OCEANS '04. MTTS/IEEE TECHNO-OCEAN '04, vol.1, no., pp.264,270 Vol.1, 9-12 Nov.

[14] Riola, J. (2011). La política de I+D en Defensa: Metas y retos tecnológicos. En Las Tecnologías de Doble Uso: La Investigación y el Desarrollo al Servicio de la Sociedad Civil y Militar (pp. 13-22). Murcia, España: Centro Universitario de la Defensa (CUD) de San Javier.

[15] Velasco, F.J.; Revestido Herrero, E.; Lopez, L.; Moyano, E., (2013) Identification for a Heading Autopilot of an Autonomous In-Scale Fast Ferry, IEEE Journal of Oceanic Engineering, vol.38, no.2, pp.263,274, April, doi: 10.1109/JOE.2012.2227175.

[16] Wasserman, L., (2006). All of Nonparametric Statistics. 